\title{
Contribution à l'évaluation du niveau de fertilité des sols dans les systèmes de culture à base du coton au Bénin
}

\author{
I. AMONMIDE ${ }^{1,2^{*}}$, G. DAGBENONBAKIN ${ }^{1}$, C. E. AGBANGBA ${ }^{1}$ et P. AKPONIKPE ${ }^{2}$ \\ ${ }^{I}$ Centre de Recherches Agricoles Coton et Fibres (CRA-CF)/Institut National des Recherches Agricoles du \\ Bénin (INRAB), 01 BP 175 Cotonou, Bénin. \\ ${ }^{2}$ Département de l'Aménagement des Ressources Naturelles, Laboratoire d'Hydrolique et de Modélisation de \\ l'Environnement (HydroModE-Lab), Université de Parakou, Bénin 03 BP: 351 Université, Parakou, Benin. \\ *Auteur correspondant ; E-mail : amonmide2018@yahoo.com ; Tél: +22997794507/95845541
}

\section{RESUME}

Le coton est la principale culture de rente au Bénin. L'accroissement continu des superficies cultivées a entraîné la sédentarisation des cultures avec pour corollaire la dégradation des sols et la baisse des rendements. L'objectif de l'étude est d'évaluer le niveau de fertilité des sols des bassins cotonniers du Bénin. Ainsi, 30 échantillons de sol ont été prélevés auprès de 30 producteurs de coton. Les résultats ont montré des sols sablo-limoneux avec un taux d'argile de 7,38 à 12,65\%. La teneur en azote varie de 0,055 à $0,076 \%$ avec un $\mathrm{pH}$ de 5,7 à 6,5. Le taux de matière organique est compris entre 1,20 à 1,52\%. La somme des cations échangeables varie de 3,98 à $6,98 \mathrm{cmol}^{+} / \mathrm{kg}$ et la capacité d'échange cationique (CEC) entre 5,35 à 7,91 $\mathrm{cmol}^{+} / \mathrm{kg}$. Le phosphore assimilable est compris entre 5 et $13 \mathrm{cmol}^{+} / \mathrm{kg}$. En définitive, les sols présentent de bonnes propriétés physiques, de piètres caractéristiques chimiques et par conséquent un faible niveau de fertilité, ce qui compromet la durabilité du système de production. L'application des amendements organiques et minéraux est donc indispensable pour le relèvement du $\mathrm{pH}$ et de la teneur en matière organique de ces sols. (C) 2019 International Formulae Group. All rights reserved

Mots clés: Sédentarisation agricole, fertilité des sols, matière organique.

\section{Contribution to soil fertility level assessment in cotton-based cropping systems in Benin}

\begin{abstract}
Cotton is the main cash crop cultivated in Benin. The continuous increase in cultivated areas has led to more sedentary agricultural system, followed by soil degradation and crop yield decline. This study aimed to assess soil fertility grade in the cotton belt of Benin. Thirty soil samples were collected, each from a cotton farm, for thorough physical and chemical analyses. Results showed that soil texture is mostly sandy-loam, with only 7.38-12.65\% clay. Sand percentage ranged from 60 to $80 \%$. Nitrogen $(\mathrm{N})$ content varied from 0.055 to $0.076 \%$, while soil pH was about 5.7-6.5. Soil Organic Matter (SOM) content was between 1.20 and $1.52 \%$. The sum of the exchangeable cations ranged from 3.98 to $6.98 \mathrm{cmol}^{+} / \mathrm{kg}$ and the cation exchange capacity (CEC) ranged from 5.35 to $7.91 \mathrm{cmol}^{+} / \mathrm{kg}$. Available Phosphorus (P) content was estimated at $5-13 \mathrm{cmol}^{+} / \mathrm{kg}$. By and large, the soils surveyed showed good physical properties, but they were chemically poor. Therefore, the inherent fertility level was low, which put their sustainable use at risk. The main target is to raise the $\mathrm{pH}$ and SOM content, through organic and mineral amendments.
\end{abstract}

(C) 2019 International Formulae Group. All rights reserved

Keywords: Sedentary agriculture, soil fertility, organic matter. 


\section{INTRODUCTION}

Dans la plupart des pays de l'Afrique de l'Ouest, la culture du coton occupe une place prépondérante sur les plans économique et social (Aho et Capo-Chichi, 2017). Au Bénin, le coton représente près de $30 \%$ des exportations et contribue, en termes de valeur ajoutée, pour $7 \%$ à la formation du Produit Intérieur Brut (PIB) (Banque Mondiale, 2016). Le coton constitue également l'activité économique principale pour plus de 300000 ménages et procure un revenu monétaire près de 2,5 millions de personnes; soit $25 \%$ de la population (Banque Mondiale, 2016). Le coton est cultivé par environ un tiers des paysans au Bénin et occupe environ 20\% des superficies cultivées (Ton, 2004).

Dans les grandes zones de production cotonnière, le coton occupe plus de $60 \%$ des emblavures à l'échelle de l'exploitation. Si au démarrage de la culture du coton au Bénin vers les années 1960, les sols étaient encore fertiles avec un système itinérant sur brûlis et des jachères de longue durée, de 1960 à nos jours, le système agraire a beaucoup évolué passant du brûlis avec jachère à une agriculture sédentaire basée sur la gestion extensive des résidus de récolte (Igué et al., 2008). Un tel système a engendré la dégradation des sols et surtout la diminution drastique du taux de matière organique et la baisse des rendements des cultures (Coulibaly et al., 2012 ; Pouya et al., 2013). Par ailleurs, l'agriculture à faible utilisation d'engrais minéraux (Batamoussi et al., 2015) ajoutée à la non valorisation des résidus de récolte, ont contribué à la perte de nutriments dans les bassins cotonniers. Sur le long terme, il y a une tendance à la baisse des rendements de l'ordre de $30 \%$ sur 20 ans, soit $11 \mathrm{~kg} / \mathrm{ha} / \mathrm{an}$ (Bassala et al., 2008). Des études menées sur l'évolution de la fertilité des sols au NordCameroun dans les bassins cotonniers (M'Biandoun et Douzet, 2000 ; Bassala et al., 2008) ont mis en évidence la baisse des teneurs en azote du sol et l'insuffisance du phosphore disponible, $\mathrm{du}$ potassium échangeable et du magnésium. L'exploitation continue des sols entraîne une dégradation rapide de leur fertilité qui se traduit par une baisse des rendements des cultures (Annabi et al., 2009 ; Koulibaly et al., 2010). Au nord de la Tunisie, les résultats obtenus par Annabi et al. (2009) ont montré que les teneurs en carbone organique total (COT) et en azote total (Ntotal) sont en étroite relation avec le type d'occupation du sol. Les sols forestiers contiennent $2,4 \%$ de COT et $0,21 \%$ de Ntotal contre 1,4 et $0,14 \%$ de COT et Ntotal, respectivement pour les sols cultivés. Dans les systèmes de culture à base du coton, la durabilité semble reposer principalement à la fois sur la fertilisation et sur les techniques de gestion rationnelle de la fertilité (Oumarou, 20).

Il a été observé dans les bassins cotonniers du Centre et du Nord-Bénin, des taux de matière organique largement inférieurs à $2 \%$, de faibles valeurs de CEC $(5,15$ à 6,75$)$ et de $\mathrm{pH}$ acide $(5,2$ à 5,7$)$ (Igué et al., 2013 ; Igué et al., 2016). Dans le bassinversant de l'Ouémé supérieur (partie septentrionale du pays), pour une estimation du bilan partiel pour la culture du maïs sur une période de 12 ans (1987-1999), le déficit minéral annuel qui était de $17 \mathrm{~kg} \mathrm{~N} / \mathrm{ha}, 3 \mathrm{~kg}$ $\mathrm{P} /$ ha et $7 \mathrm{~kg} \mathrm{~K} /$ ha en 1987 , est passé à $23 \mathrm{~kg}$ $\mathrm{N} / \mathrm{ha}, 14 \mathrm{~kg} \mathrm{P} / \mathrm{ha}$ et $21 \mathrm{~kg} \mathrm{~K} / \mathrm{ha}$ en 2000 (Dagbenonbakin et al., 2002). Ce bilan négatif était plus sévère en 2002 dans une rotation igname-coton-maïs-arachide-sorgho avec notamment un déficit annuel de $37 \mathrm{~kg} \mathrm{~N} / \mathrm{ha}, 8$ $\mathrm{kg} \mathrm{P} / \mathrm{ha}, 35 \mathrm{~kg} \mathrm{~K} / \mathrm{ha}$ (Dagbenonbakin, 2005). L'épuisement devient alors plus important dans le temps (Ouandaogo et al., 2016) à cause de l'exploitation des sols sans restitution des résidus de récolte avec très peu ou pas du tout d'engrais sur les cultures conduisant ainsi à une baisse de leur fertilité et par conséquent une baisse des rendements des cultures. Par ailleurs, les études de la carte de fertilité du Bénin par zone agro-écologique conduites par Azontondé et al. (2009) ont montré des niveaux de fertilité très bas et bas dans les régions de Banikoara et de Kandi. Dans ces zones de grande production cotonnière, les sols sont dégradés sur les plans chimique, physique et biologique. Des limitations sévères ont été observées en matière organique et en nutriments. La 
production étant déterminée par ces facteurs et les relations existant entre eux, il est essentiel de maintenir le taux de matière organique et des éléments minéraux à des proportions pouvant garantir une bonne productivité des terres tout en préservant le sol contre toute forme de dégradation. Mais aucune de ces études n'a pris en compte, au même moment, tous les bassins cotonniers du Bénin. Le coton étant la principale culture de rente et la culture locomotive pour les cultures vivrières, il est important que la fertilité des sols soit bien suivie et bien gérée dans les zones où il est produit. Avec les problèmes de changement climatique, où les pluies sont mal réparties, il devient urgent de veiller au maintien voire l'amélioration du taux de carbone dans le sol. C'est pourquoi, il est nécessaire de faire une évaluation périodique des indicateurs de fertilité dans les grandes zones de production cotonnière afin de faire des recommandations de gestion efficiente de la précieuse ressource terre.

Le présent travail avait pour objectif d'évaluer les paramètres physico-chimiques des sols pour une gestion durable des exploitations agricoles dans les systèmes de production à base du coton.

\section{MATERIEL ET METHODES \\ Zones d'étude}

L'étude a été réalisée dans les quatre grandes zones cotonnières du Bénin (Hougni al., 2016). Dans la zone 1 (Figure 1) qui regroupe les départements de l'Alibori et de l'Atacora, l'étude a été conduite dans les communes de Kandi $\left(11^{\circ} 07^{\prime} 43^{\prime}\right.$ 'N, $\left.2^{\circ} 56^{\prime} 13^{\prime \prime} \mathrm{E}\right)$, de Banikoara $\left(11^{\circ} 18^{\prime} 00^{\prime} \mathrm{N}\right.$, $\left.2^{\circ} 26^{\prime} 00^{\prime \prime} \mathrm{E}\right)$ et de Kérou $\left(10^{\circ} 49^{\prime} 30^{\prime \prime} \mathrm{N}\right.$, $\left.2^{\circ} 06^{\prime} 34^{\prime \prime} \mathrm{E}\right)$. Dans la zone 2 qui regroupe le département du Borgou et de la Donga, l'étude a été conduite dans la commune de Sinendé $\left(10^{\circ} 20^{\prime} 41^{\prime \prime} \mathrm{N}, 2^{\circ} 22^{\prime} 45^{\prime \prime} \mathrm{E}\right)$. Dans la zone 3 qui regroupe le département des Collines, l'étude a été conduite dans la commune de Savalou $\left(7^{\circ} 55^{\prime} 50^{\prime}\right.$ 'N, $\left.1^{\circ} 58^{\prime} 31^{\prime \prime} \mathrm{\prime}\right)$. Dans la zone 4 qui regroupe les départements du Zou, du Couffo et du Plateau, l'étude a été conduite dans la commune de Djidja (1³9'0'N', 1'13'0'’E).
Les deux premières zones sont caractérisées par un climat de type soudanosahélien (tropical humide) à régime pluviométrique unimodal à une saison des pluies (mai à octobre) et une saison sèche (novembre à avril). La pluviosité moyenne annuelle varie de 600 à $1200 \mathrm{~mm}$ avec une répartition annuelle de 30 à 70 jours.

Les deux dernières zones sont caractérisées par un climat de type soudanoguinéen (tropical humide) à régime pluviométrique bimodal à deux saisons pluvieuses et à deux saisons sèches. Une grande saison des pluies (mars à juillet), une petite saison sèche en août, une petite saison des pluies (septembre à novembre) et une grande saison sèche (décembre à mars). La pluviosité varie de 700 à $1800 \mathrm{~mm}$ avec une répartition annuelle de 48 à 80 jours.

\section{Choix des zones d'étude et des producteurs}

Le choix a porté sur les grandes communes productrices du coton au niveau des quatre grandes zones de production cotonnière au Bénin (Hougni al., 2016). Au total, 30 producteurs ont été sélectionnés à raison de 5 par commune. Il s'agit des producteurs ouverts aux innovations agricoles.

\section{Echantillonnage et analyses de sols}

Trente (30) échantillons composites de sol ont été prélevés à $0-20 \mathrm{~cm}$ de profondeur et expédiés au Laboratoire des Sciences du Sol, Eaux et Environnement (LSSEE), de l'Institut Nationale des Recherches Agricoles du Bénin (INRAB) pour la détermination des caractéristiques physico-chimiques conformément aux méthodes de Tran Vin An (1978). Ces analyses ont consisté en la détermination de la granulométrie (5 fractions), réalisée par la méthode internationale modifiée par l'emploi de la pipette de Robinson, du carbone organique, déterminé par la méthode de Walkley et Black (1934); de l'azote total, déterminé par la méthode de Kjeldahl; du pHeau, à l'aide d'un pH-mètre avec $(1 / 2,5)$ comme ratio sol-eau; du phosphore assimilable, par la méthode Bray1; de la capacité d'échange cationique (CEC), par l'extration au $\mathrm{KCl} 10 \%$ puis 
distillation par la méthode de Kjeldahl; des cations échangeables ( $\mathrm{Ca}, \mathrm{Mg}$ et $\mathrm{K})$, dosés au Spectrophotométrie à Absorption Atomique et l'aluminium échangeable.

Méthode d'évaluation des niveaux de fertilité des sols

La fertilité des sols étudiés a été définie à partir des classes de fertilité chimiques (Tableau 1).

Classement des niveaux de fertilité chimique des sols

Classe I, niveau de fertilité élevé : Les sols sont de cette classe lorsque les caractéristiques ne présentent pas ou présentent seulement de faibles limitations.
Classe II, niveau de fertilité moyen : Les sols sont de cette classe lorsque les caractéristiques ne présentent pas plus de 3 limitations modérées éventuellement associées à de faibles limitations.

Classe III, niveau de fertilité bas : Les sols sont de cette classe lorsque ses caractéristiques présentent plus de 3 limitations modérées associées à une seule limitation sévère.

Classe IV, niveau de fertilité très bas : Les sols sont de cette classe lorsque ses caractéristiques présentent plus d'une limitation sévère.

Tableau 1 : Critères d'évaluation des classes de fertilité des sols.

\begin{tabular}{llllll}
\hline Caractéristiques & \multicolumn{5}{c}{ Niveau de fertilité } \\
\cline { 2 - 6 } & $\begin{array}{l}\text { Très élevé (sans } \\
\text { limitations) }\end{array}$ & $\begin{array}{l}\text { Elevé } \\
\text { (limitation } \\
\text { faible) }\end{array}$ & $\begin{array}{l}\text { moyen } \\
\text { (limitations } \\
\text { moyennes) }\end{array}$ & $\begin{array}{l}\text { bas } \\
\text { (limitations } \\
\text { sévères) }\end{array}$ & $\begin{array}{l}\text { très bas } \\
\text { (limitations } \\
\text { très sévères) }\end{array}$ \\
\cline { 2 - 6 } & Degré 0 & Degré 1 & Degré 2 & Degré 3 & Degré 4 \\
\hline $\mathrm{MO}(\%)$ & $>2$ & $2-1,5$ & $1,5-1$ & $1-0,5$ & $<0,5$ \\
\hline $\mathrm{N}(\%)$ & $>0,08$ & $0,08-0,06$ & $0,06-0,045$ & $0,045-0,03$ & $<0,03$ \\
\hline $\mathrm{Pass}\left(\mathrm{cmol}^{+} / \mathrm{kg}\right)$ & $>20$ & $20-15$ & $15-10$ & $10-5$ & $<5$ \\
\hline $\mathrm{K}^{+}\left(\mathrm{cmol}^{+} / \mathrm{kg}\right)$ & $>0,4$ & $0,4-0,3$ & $0,3-0,2$ & $0,2-0,1$ & $<0,1$ \\
\hline $\mathrm{Somme} \mathrm{des} \mathrm{cations}$ & $>10$ & $10-7,5$ & $7,5-5$ & $5-2$ & $<2$ \\
$\left(\mathrm{cmol}^{+} / \mathrm{kg}\right)$ & & & & & \\
\hline $\mathrm{V}(\%)$ & $>60$ & $60-50$ & $50-30$ & $30-15$ & $<15$ \\
\hline $\mathrm{CEC}\left(\mathrm{cmol}^{+} / \mathrm{kg}\right)$ & $>25$ & $25-15$ & $15-10$ & $10-5$ & $<5$ \\
\hline $\mathrm{pH}$ & $5,5-6,5$ & $5,5-6,0$ & $5,5-5,3$ & $5,3-5,2$ & $<5,2$ \\
& $6,5-8,2$ & $6,5-7,8$ & $7,8-8,3$ & $8,3-8,5$ & $>8,5$ \\
\hline
\end{tabular}

Sources : Dabin (1956); Sys (1993). 


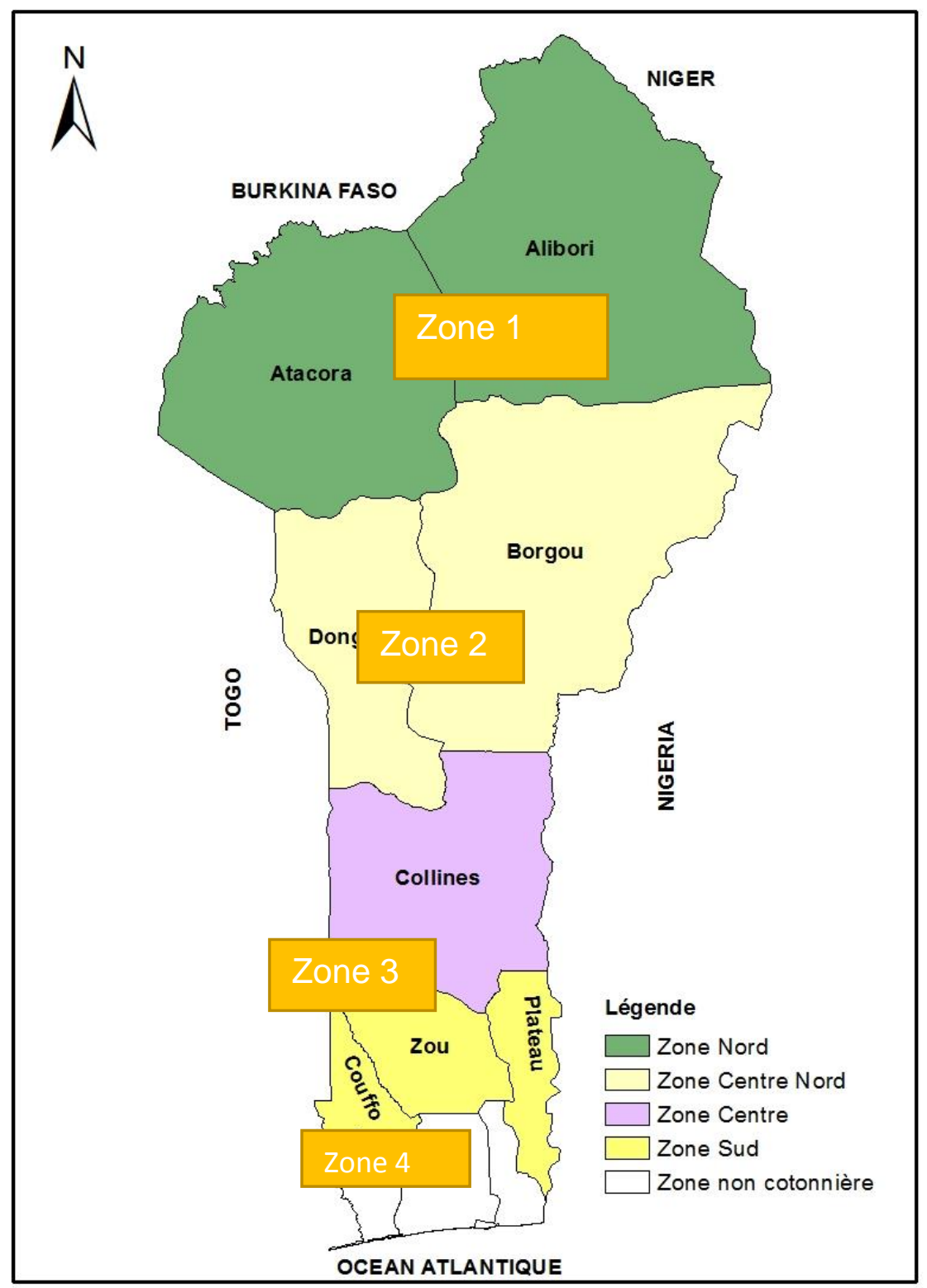

Figure 1 : Carte administrative du Bénin montrant les zones d'étude.

\section{RESULTATS}

\section{Granulométrie}

Les sols sont sablo-limoneux. Le taux de limon varie de 13 à $25 \%$. Celui d'argile varie de 7,38 à $12,65 \%$. Le taux de sable est compris entre 60 et $80 \%$. Les proportions d'argile sont globalement faibles et inférieures à la norme moyenne (Tableau 2) au niveau des six communes. Cependant, les sols de Kandi, Banikoara et Kérou sont plus pourvus en argile que ceux de Sinendé, Savalou et de Djidja. Hormis la faiblesse du taux d'argile dans ces trois dernières communes, la granulométrie est globalement bonne.

\section{Taux moyen en azote total et en matière organique}

Le taux moyen en azote total varie de 0,055 à $0,076 \%$ contre la norme qui est de 0,1 à $0,15 \%$. Le taux le plus élevé a été observé 
dans la commune de Kandi (Tableau 2). Pour l'ensemble des communes, $27 \%$ des exploitations ont présenté un très faible taux, $67 \%$, un faible taux et un taux moyen, seulement dans une proportion de 6\%.

Les taux moyen de matière organique est très faible et varie de 1,20 à 1,52\% contre la norme de 2 à $3 \%$. Le taux le plus élevé a été observé dans la commune de Kandi (Tableau 2). Les sols sont largement pauvres en matière organique $(73 \%)$, très pauvres $(17 \%)$ et moyen $(10 \%)$.

\section{Cations échangeables \\ $\checkmark$ Calcium}

La concentration moyenne du calcium échangeable varie de 3,09 à $4,93 \mathrm{cmol}^{+} / \mathrm{kg}$ contre la norme de 2,3 à $3,5 \mathrm{cmol}^{+} / \mathrm{kg}$. A l'exception de la commune de Kérou, les concentrations de calcium échangeable sont très variables à l'intérieur d'une même commune. Les sols des communes de Kérou, de Banikoara et de Kandi sont plus pourvus en calcium échangeable que ceux des trois autres communes (Tableau 2).

\section{$\checkmark$ Magnésium}

$\mathrm{La}$ concentration moyenne du magnésium échangeable a varié de 0,65 à 1,71 $\mathrm{cmol}^{+} / \mathrm{kg}$ contre la norme de 1 à $1,5 \mathrm{cmol}^{+} / \mathrm{kg}$. Seule la commune de Kandi a enregistré des concentrations qui ont atteint le seuil de la moyenne. Dans les autres communes, ce seuil moyen n'est pas atteint. Diverses classes de concentration ont été identifiées. Plus de la moitié (53\%) des sols sont pauvres en magnésium, $10 \%$ sont très pauvres, $17 \%$ sont moyens, $13 \%$ sont riches et $7 \%$ sont très riches.

\section{$\checkmark$ Potassium}

La concentration moyenne du potassium échangeable a varié de 0,13 à 0,23 $\mathrm{cmol}^{+} / \mathrm{kg}$ contre la norme de 0,2 à 0,4 $\mathrm{cmol}^{+} / \mathrm{kg}$. Dans les communes de Kérou, Savalou et Djidja, le seuil moyen n'est pas atteint. Le potassium échangeable est très bas dans ces trois localités. Ainsi, 7\% des sols sont très pauvres, $70 \%$ pauvres et $33 \%$ sont moyens.

\section{$\checkmark$ Sodium}

La concentration moyenne de sodium échangeable a varié de 0,07 à $0,12 \mathrm{cmol}^{+} / \mathrm{kg}$ contre la norme de 0,3 à $0,7 \mathrm{cmol}^{+} / \mathrm{kg}$. La classification en cet élément a montré des sols très pauvres $(80 \%)$, pauvres $(17 \%)$ et moyens (3\%).

\section{Somme des cations échangeables}

La concentration en cations échangeables a varié de 3,98 à $6,98 \mathrm{cmol}^{+} / \mathrm{kg}$ contre la norme de 5 à $10 \mathrm{cmol}^{+} / \mathrm{kg}$. Le seuil moyen est atteint dans les communes de Kandi, Banikoara et Kérou. Dans les trois autres communes, les taux moyens sont en dessous de la moyenne. Ainsi, 7\%, 57\%, 27\% et $10 \%$ des sols sont respectivement très pauvres, pauvres, moyens et riches en cations échangeables.

pH et CEC

Le $\mathrm{pH}$ a varié de 5,7 à 6,5 contre la référence de 6,5 à 7,5. Les bas $\mathrm{pH}$ sont surtout observés dans les communes de Savalou et de Djidja (Tableau 2). Dans l'ensemble, le pH est acide à peu acide dans $83 \%$ des cas et neutre seulement dans $17 \%$ des cas.

La CEC a varié de 5,35 et 7,91 $\mathrm{cmol}^{+} / \mathrm{kg}$ contre la norme de 10 à $25 \mathrm{cmol}^{+} / \mathrm{kg}$. Les CEC moyens par commune sont faibles, et sont en dessous de la moyenne (Tableau 2). Ainsi, $40 \%$ des sols ont des CEC très pauvres, $47 \%$ sont pauvres et $13 \%$ seulement sont moyens.

\section{Taux de saturation des cations}

Le taux de saturation a varié de 62 à $91,8 \%$ contre la norme variant de 40 à $60 \%$. Par rapport aux charges négatives disponibles sur le complexe argilo-humique, la plupart des sols sont bien saturés en cations échangeables. La classification donne 3\% des sols désaturés, $10 \%, 53 \%$ et $33 \%$ sont respectivement, moyens, bien et très bien saturés en cations échangeables.

\section{Phosphore assimilable}

Les concentrations en phosphore assimilable ont varié de 5 à $13 \mathrm{cmol}^{+} / \mathrm{kg}$ contre la norme de 10 à $15 \mathrm{cmol}^{+} / \mathrm{kg}$. Les concentrations appartenant à la classe moyenne sont enregistrées seulement dans les communes de Sinendé et de Savalou. Cependant, une grande variabilité a été observée à Sinendé (Tableau 2). Ainsi, 50\%, $30 \%, 10 \%, 3 \%$ et $7 \%$ des sols sont 
respectivement très pauvres, pauvres, moyens, riches et très riches en phosphore assimilable.

\section{Influence de la matière organique sur la CEC et le potassium échangeable et relation entre l'argile et la capacité d'échange cationique (CEC)}

Une relation linéaire a été observée entre le taux de matière organique et la CEC $\left(\mathrm{R}^{2}=0,44\right.$; Figure 2). Plus la teneur en matière organique du sol augmente, la CEC devient importante. Le seuil moyen de la CEC est atteint lorsque le taux de matière organique est au moins égal à 1,5\%. La même tendance a été observée entre le taux de matière organique et la concentration en potassium échangeable $\left(\mathrm{R}^{2}=0,33\right.$; Figure 3$)$. Une relation a été également observée entre le taux d'argile et la CEC (Figure 4).

\section{Evaluation du niveau de fertilité des sols}

La plupart des paramètres physicochimiques sont très bas (Tableau 3). L'ensemble des sols présentent plus de deux limitations sévères (Tableau 4). La matière organique (MO), la capacité d'échange cationique (CEC), la somme des cations échangeables (SCE) et le phosphore assimilable sont limitatifs pour l'ensemble des communes. La limitation est moyenne pour la MO alors qu'elle est sévère au niveau de la CEC et du phosphore assimilable. Si la teneur moyenne en azote total (Ntotal) n'est pas limitative dans la commune de Kandi, elle a une limitation moyenne dans les autres communes. Le potassium échangeable présente trois limitations sévères, deux limitations moyennes et une faible. Par rapport aux cations échangeables, trois communes présentent une limitation sévère. $\mathrm{Au}$ niveau des trois autres communes, la limitation est moyenne. Par ailleurs, en dehors de la commune de Banikoara où le $\mathrm{pH}$ est proche de la neutralité, les $\mathrm{pH}$ observés dans les autres localités sont relativement bas. Cependant, l'ensemble des $\mathrm{pH}$ ne sont pas limitatifs pour un développement moyen du cotonnier. Tous les sols appartiennent à la classe IV avec un très bas niveau de fertilité.

Tableau 2 : Caractéristiques physico-chimiques des sols des différentes localités.

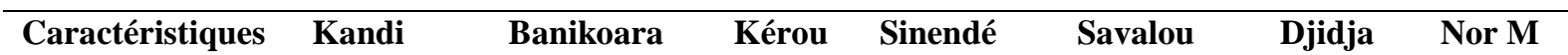

\begin{tabular}{|c|c|c|c|c|c|c|c|}
\hline \multirow[t]{2}{*}{ Argiles\% } & 12,65 & $11,65(6,21)$ & 11,88 & 8,95 & 7,14 & 7,38 & $25-30$ \\
\hline & $(4,52)$ & & $(5,68)$ & $(5,39)$ & $(3,01)$ & $(1,95)$ & \\
\hline \multirow[t]{2}{*}{ Limon fin\% } & $7,99(2,77)$ & $7,86(3,16)$ & 8,98 & 11,36 & $5,67(4,27)$ & 6,07 & \\
\hline & & & $(2,92)$ & $(8,58)$ & & $(2,58)$ & \\
\hline \multirow[t]{2}{*}{ Limon grossier\% } & 11,02 & $14,11(6,55)$ & 12,78 & 14,82 & $8,07(4,47)$ & 8,26 & \\
\hline & $(4,25)$ & & $(2,23)$ & $(9,51)$ & & $(2,74)$ & \\
\hline \multirow[t]{2}{*}{ Sable fin $\%$} & 34,74 & $36,40(10,13)$ & 32,93 & 31,19 & 49,53 & 36,55 & \\
\hline & $(8,60)$ & & $(4,15)$ & $(6,74)$ & $(14,81)$ & $(5,57)$ & \\
\hline \multirow[t]{2}{*}{ Sable Grossier\% } & 33,59 & $29,98(12,30)$ & 33,42 & 33,67 & 49,53 & 41,74 & \\
\hline & $(8,35)$ & & $(11,43)$ & $(22,27)$ & $(14,81)$ & $(9,99)$ & \\
\hline \multirow[t]{2}{*}{$\mathrm{N} \%$} & 0,076 & $0,059(0,01)$ & 0,061 & 0,064 & 0,058 & 0,055 & 0,1 à \\
\hline & $(0,03)$ & & $(0,01)$ & $(0,02)$ & $(0,02)$ & $(0,00)$ & 0,15 \\
\hline \multirow[t]{2}{*}{$\mathrm{C} \%$} & 0,88 & $0,70(0,14)$ & 0,81 & 0,78 & 0,69 & 0,76 & 2 à 3 \\
\hline & $(0,36)$ & & $(0,21)$ & $(0,41)$ & $(0,34)$ & $(0,07)$ & \\
\hline \multirow[t]{2}{*}{$\mathrm{MO} \%$} & 1,52 & $1,20(0,25)$ & 1,34 & $1,35(0,71)$ & $1,20(0,59)$ & 1,30 & 2 à 3 \\
\hline & $(0,62)$ & & $(0,35)$ & & & $(0,12)$ & \\
\hline
\end{tabular}


I. AMONMIDE et al. / Int. J. Biol. Chem. Sci. 13(3): 1846-1860, 2019

\begin{tabular}{llllllll}
\hline $\mathrm{pH}_{\text {eau }}(1 / 2,5)$ & 6,1 & $6,5(0,6)$ & 6,1 & $6,1(0,8)$ & 5,8 & $5,7(0,4)$ & 6,5 à \\
& $(0,2)$ & & $(0,3)$ & & $(0,6)$ & & 7,5 \\
\hline $\mathrm{Ca}^{2+}(\mathrm{Cmol}+\mathrm{kg})$ & $4,93(4,08)$ & $4,22(2,49)$ & 3,89 & $3,25(1,94)$ & 3,21 & 3,09 & 2,3 à \\
& & & $(2,15)$ & & $(2,22)$ & $(0,50)$ & 3,5 \\
\hline $\mathrm{Mg}^{2+}(\mathrm{Cmol}+/ \mathrm{kg})$ & 1,71 & $0,98(0,70)$ & 1,76 & $0,98(0,47)$ & $0,69(0,54)$ & 0,65 & 1 à 1,5 \\
& $(1,02)$ & & $(1,21)$ & & & $(0,06)$ & \\
\hline $\mathrm{K}^{+}\left(\mathrm{Cmol}^{+} / \mathrm{kg}\right)$ & $0,25(0,15)$ & $0,16(0,05)$ & 0,14 & $0,23(0,10)$ & $0,12(0,03)$ & 0,13 & 0,2 à \\
& & & $(0,04)$ & & & $(0,01)$ & 0,4 \\
\hline $\mathrm{Na}^{+} \mathrm{Cmol}^{+} / \mathrm{kg}$ & 0,07 & $0,10(0,02)$ & 0,07 & 0,12 & $0,07(0,01)$ & 0,09 & 0,3 à \\
& $(0,00)$ & & $(0,01)$ & $(0,13)$ & & $(0,03)$ & 0,7 \\
\hline $\mathrm{SCE} \mathrm{Cmol}^{+} / \mathrm{kg}$ & 6,98 & $5,13(3,14)$ & 5,88 & 4,53 & $4,10(2,77)$ & 3,98 & 5 à 10 \\
& $(5,18$ & & $(3,38)$ & $(2,46)$ & & $(0,53)$ & \\
\hline $\mathrm{CEC} \mathrm{Cmol}^{+} / \mathrm{kg}$ & $7,91(4,74)$ & $7,25(3,68)$ & 6,18 & $5,35(2,62)$ & $6,76(2,43)$ & 6,12 & 10 à 25 \\
& & & $(3,00)$ & & & $(2,01)$ & \\
\hline$\% \mathrm{~V}=\mathrm{S} / \mathrm{T}^{*} 100$ & 81,8 & $71(19,96)$ & 91,8 & 81,4 & $62(30,51)$ & 68,8 & 40 à 60 \\
& $(19,72)$ & & $(10,83)$ & $(15,77)$ & & $(16,18)$ & \\
\hline $\mathrm{P}_{\text {ass. Bray l } \mathrm{ppm}}$ & $5(3,9)$ & $5,2(3,3)$ & 2,4 & $12,8(11,8)$ & 7 & $4,6(1,6)$ & 10 à 15 \\
& & & $(1,1)$ & & $(5,8)$ & & \\
\hline
\end{tabular}

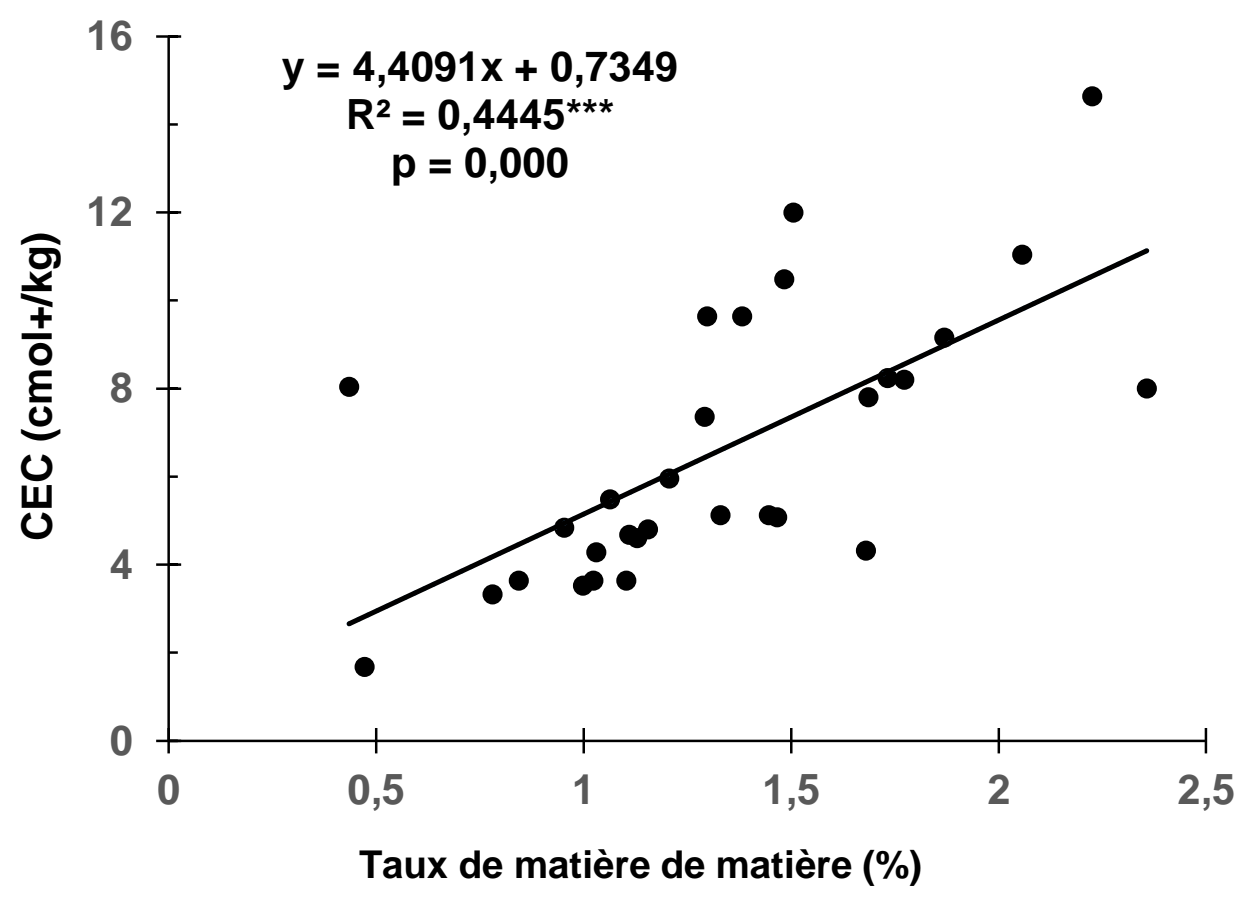

Figure 2 : Effet de la matière organique sur la CEC. 


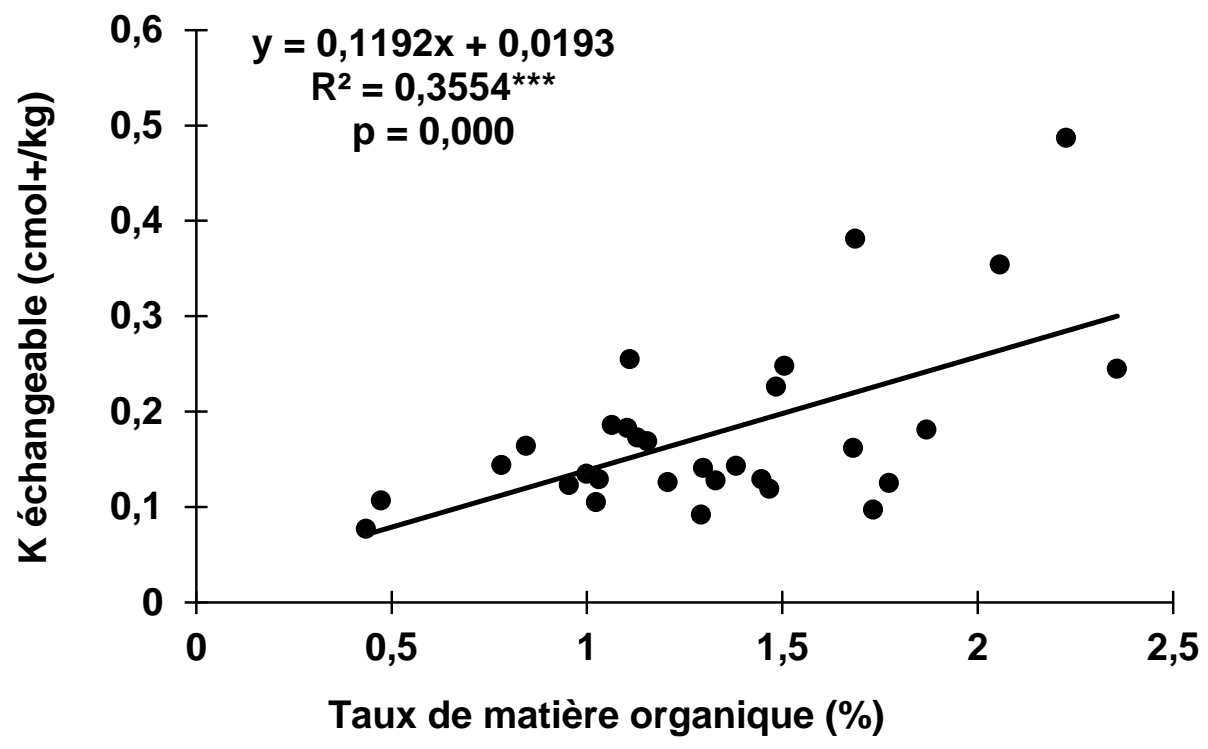

Figure 3 : Effet du taux de la MO sur la concentration en potassium échangeable.

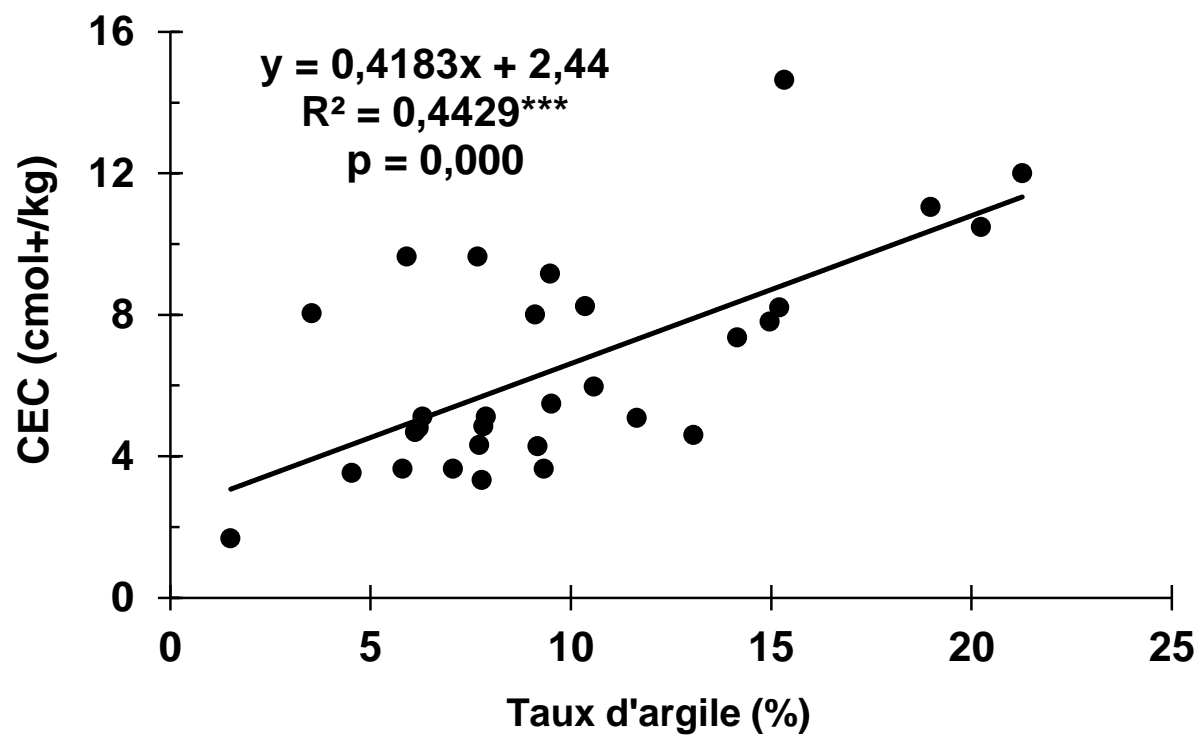

Figure 4 : Effet de l'argile sur la CEC.

Tableau 3 : Identification des paramètres physico-chimiques limitatifs.

\begin{tabular}{lllllll}
\hline Caractéristiques & Kandi & Banikoara & Kérou & Sinendé & Savalou & Djidja \\
\hline $\mathrm{MO}(\%)$ & 1,52 & 1,2 & 1,34 & 1,35 & 1,20 & 1,3 \\
\hline $\mathrm{N}(\%)$ & 0,08 & 0,06 & 0,06 & 0,06 & 0,06 & 0,05 \\
\hline Pass $\left(\mathrm{cmol}^{+} / \mathrm{kg}\right)$ & 5 & 5,2 & 2,4 & 12,8 & 7 & 4,6 \\
\hline $\mathrm{K}^{+}\left(\mathrm{cmol}^{+} / \mathrm{kg}\right)$ & 0,3 & 0,2 & 0,1 & 0,2 & 0,1 & 0,1 \\
\hline
\end{tabular}




\begin{tabular}{lllllll}
\hline $\mathrm{SCE}\left(\mathrm{cmol}^{+} / \mathrm{kg}\right)$ & 6,9 & 5,1 & 5,9 & 4,5 & 4,1 & 3,9 \\
\hline $\mathrm{CEC}\left(\mathrm{cmol}^{+} / \mathrm{kg}\right)$ & 7,9 & 7,2 & 6,2 & 5,3 & 6,8 & 6,1 \\
\hline $\mathrm{V}(\%)$ & 81,8 & 71 & 91,8 & 81,4 & 62 & 68,8 \\
\hline $\mathrm{pH}$ & 6,1 & 6,5 & 6,1 & 6,1 & 5,8 & 5,7 \\
\hline Facteurs limitants & MO, P, K, & MO, N, P & MO, N, P & MO, N, P & MO, N, P & MO, N, P \\
& SCE, CEC & K, SCE, & K, SCE & K, SCE & K, SCE & K, SCE \\
& & CEC & CEC & CEC & CEC & CEC \\
\hline
\end{tabular}

Tableau 4 : Degré de limitation des paramètres physico-chimiques et niveau de fertilité des sols.

\begin{tabular}{|c|c|c|c|c|c|c|}
\hline Caractéristiques & Kandi & Banikoara & Kérou & Sinendé & Savalou & Djidja \\
\hline $\mathrm{MO}(\%)$ & $\begin{array}{l}\text { Limitation } \\
\text { faible }\end{array}$ & $\begin{array}{l}\text { Limitation } \\
\text { moyenne }\end{array}$ & $\begin{array}{l}\text { Limitation } \\
\text { moyenne }\end{array}$ & $\begin{array}{l}\text { Limitation } \\
\text { moyenne }\end{array}$ & $\begin{array}{l}\text { Limitation } \\
\text { moyenne }\end{array}$ & $\begin{array}{l}\text { Limitation } \\
\text { moyenne }\end{array}$ \\
\hline $\mathrm{N}(\%)$ & $\begin{array}{l}\text { Sans } \\
\text { limitation } \\
\end{array}$ & $\begin{array}{l}\text { Limitation } \\
\text { moyenne }\end{array}$ & $\begin{array}{l}\text { Limitation } \\
\text { moyenne }\end{array}$ & $\begin{array}{l}\text { Limitation } \\
\text { moyenne }\end{array}$ & $\begin{array}{l}\text { Limitation } \\
\text { moyenne }\end{array}$ & $\begin{array}{l}\text { Limitation } \\
\text { moyenne }\end{array}$ \\
\hline Pass $\left(\mathrm{cmol}^{+} / \mathrm{kg}\right)$ & $\begin{array}{l}\text { Limitation } \\
\text { sévère }\end{array}$ & $\begin{array}{l}\text { Limitation } \\
\text { sévère }\end{array}$ & $\begin{array}{l}\text { Limitation } \\
\text { sévère }\end{array}$ & $\begin{array}{l}\text { Limitation } \\
\text { moyenne }\end{array}$ & $\begin{array}{l}\text { Limitation } \\
\text { sévère }\end{array}$ & $\begin{array}{l}\text { Limitation } \\
\text { sévère }\end{array}$ \\
\hline $\mathrm{K}^{+}\left(\mathrm{cmol}^{+} / \mathrm{kg}\right)$ & $\begin{array}{l}\text { Limitation } \\
\text { faible }\end{array}$ & $\begin{array}{l}\text { Limitation } \\
\text { moyenne }\end{array}$ & $\begin{array}{l}\text { Limitation } \\
\text { sévère }\end{array}$ & $\begin{array}{l}\text { Limitation } \\
\text { moyenne }\end{array}$ & $\begin{array}{l}\text { Limitation } \\
\text { sévère }\end{array}$ & $\begin{array}{l}\text { Limitation } \\
\text { sévère }\end{array}$ \\
\hline $\mathrm{SCE}\left(\mathrm{cmol}^{+} / \mathrm{kg}\right)$ & $\begin{array}{l}\text { Limitation } \\
\text { moyenne }\end{array}$ & $\begin{array}{l}\text { Limitation } \\
\text { moyenne }\end{array}$ & $\begin{array}{l}\text { Limitation } \\
\text { moyenne }\end{array}$ & $\begin{array}{l}\text { Limitation } \\
\text { sévère }\end{array}$ & $\begin{array}{l}\text { Limitation } \\
\text { sévère }\end{array}$ & $\begin{array}{l}\text { Limitation } \\
\text { sévère }\end{array}$ \\
\hline $\mathrm{CEC}\left(\mathrm{cmol}^{+} / \mathrm{kg}\right)$ & $\begin{array}{l}\text { Limitation } \\
\text { sévère }\end{array}$ & $\begin{array}{l}\text { Limitation } \\
\text { sévère }\end{array}$ & $\begin{array}{l}\text { Limitation } \\
\text { sévère }\end{array}$ & $\begin{array}{l}\text { Limitation } \\
\text { sévère }\end{array}$ & $\begin{array}{l}\text { Limitation } \\
\text { sévère }\end{array}$ & $\begin{array}{l}\text { Limitation } \\
\text { sévère }\end{array}$ \\
\hline $\mathrm{V}(\%)$ & Sans & Sans limitation & Sans & Sans & Sans & Sans \\
\hline & limitation & & limitation & limitation & limitation & limitation \\
\hline $\mathrm{pH}$ & Sans & Sans limitation & Sans & Sans & Sans & Sans \\
\hline Facteurs limitants & $\frac{\text { limitation }}{\mathrm{MO}, \mathrm{P}, \mathrm{K}}$ & $\mathrm{MO}, \mathrm{N}, \mathrm{P}$ & $\frac{\text { limitation }}{\mathrm{MO}, \mathrm{N}, \mathrm{P}}$ & $\frac{\text { limitation }}{\mathrm{MO}, \mathrm{N}, \mathrm{P}}$ & $\frac{\text { limitation }}{\mathrm{MO}, \mathrm{N}, \mathrm{P}}$ & $\frac{\text { limitation }}{\mathrm{MO}, \mathrm{N}, \mathrm{P}}$ \\
\hline & SCE, CEC & $\mathrm{K}, \mathrm{SCE}, \mathrm{CEC}$ & $\mathrm{K}, \mathrm{SCE}$ & $\mathrm{K}, \mathrm{SCE}$ & $\mathrm{K}, \mathrm{SCE}$ & $\mathrm{K}, \mathrm{SCE}$ \\
\hline & & & CEC & CEC & CEC & CEC \\
\hline Classe de sol & IV & IV & IV & IV & IV & IV \\
\hline Niveau de fertilité & Très bas & Très bas & Très bas & Très bas & Très bas & Très bas \\
\hline
\end{tabular}

\section{DISCUSSION}

\section{Granulométrie et matière organique}

Les proportions de sable et de limon dominent largement celle de l'argile dans toutes les localités. Les sols sont de texture sablo-limoneuse avec plus de $60 \%$ de sable. Les taux d'argile sont largement inférieurs à $20 \%$; ce qui n'est pas de nature à favoriser la rétention de l'eau et des éléments nutritifs. L'argile est l'élément qui conditionne la fixation des éléments minéraux sur le complexe adsorbant. De plus, la forte proportion de sable serait liée aux effets du labour et de l'exploitation en continu des terres qui provoquent le lessivage des particules fines (Koulibaly et al., 2014). Les taux moyens de matière organique variant de 1,20 à $1,52 \%$ sont faibles. Toutes ces teneurs sont en dessous de la norme qui est de 2 à $3 \%$ (Mulaji et al., 2016). Ces faibles teneurs en 
matière organique dans les sols, les prédisposeraient à une acidification et à une dégradation rapide.

L'argile et la matière organique étant la base du complexe argilo-humique, leur déficience contribuerait en grande partie à la dégradation de la fertilité de ces sols (Yemefack et al., 2004).

\section{Matière organique, $\mathrm{CEC}$ et $\mathrm{pH}$}

Les valeurs de la CEC des sols des différentes localités sont très faibles. La CEC a varié de 5,35 à $7,9 \mathrm{cmol}^{+} / \mathrm{kg}$ contre la norme variant de 10 à $25 \mathrm{cmol}^{+} / \mathrm{kg}$. Elle est très pauvre à pauvre dans $87 \%$ des exploitations. Ceci serait dû à la faiblesse du taux de matière organique et d'argile observée dans les différentes localités. Les résultats ont montré que le taux d'argile et de matière organique expliquent la fixation des cations échangeables sur le complexe argilo-humique dans une proportion de $44 \%$. Ces résultats confirment ceux obtenus par Alexandre et al. (2012); Koull et Halilat (2016). En effet, ces différents auteurs ont montré que la CEC est intimement liée au taux de matière organique et au taux d'argile dans le sol. Par contre, la liaison entre le $\mathrm{pH}$ et la CEC est faible et s'explique seulement dans une proportion de $16 \%$. En effet, parmi les fractions minérales, la kaolinite, les oxydes et hydroxydes de fer et d'aluminium sont ceux qui disposent une grande partie de leur CEC dépendante du $\mathrm{pH}$. Quant à la matière organique, toutes ses charges sont variables en fonction du $\mathrm{pH} d u$ sol (Mbonigaba et al., 2009). En effet, à des valeurs faibles de $\mathrm{pH}(\mathrm{pH}<5)$, la matière organique a tendance à développer plus de charges positives et les ions $\mathrm{H}+$ issus de la déprotonation provoquent la solubilisation des hydroxydes d'aluminium présents dans le milieu et ainsi apparaissent les formes toxiques $\left[\mathrm{Al}(\mathrm{OH})^{2+}, \quad \mathrm{Al}(\mathrm{OH})^{+}, \mathrm{Al}^{3+}\right] . \quad \mathrm{Par}$ contre, dans les conditions d'acidité moyenne $(\mathrm{pH} \geq 5)$, la charge nette des colloïdes organiques dans le sol reste négative (Menzies, 2003). Dans la présente étude, la plupart des $\mathrm{pH}$ sont supérieurs à 5 , la CEC est donc influencée par le taux de matière organique. Il est important de souligner que les valeurs de CEC obtenues (Tableau 2) sont proches de la CEC seuil $\left(4 \mathrm{cmol}^{+} / \mathrm{kg}\right)$. Il y a donc urgence de développer des techniques de production locale et de valorisation de la matière organique qui permettront de réhausser le $\mathrm{pH}$ du sol pour augmenter la capacité de rétention de ces sols afin de prévenir les pertes des éléments nutritifs par lessivage, plus particulièrement les cations basiques échangeables. Ainsi, la matière organique joue un rôle déterminant dans l'amélioration de la fertilité des sols (Tchabi et al., 2012). Cependant, dans un essai d'amendement organique de longue durée, il a été montré une forte influence du $\mathrm{pH}$ sur la CEC. En effet, un $\mathrm{pH}$ élevé affecte positivement la CEC (Pernes-Debuyser et Tessier, 2002).

\section{Cations échangeables et saturation en cations}

Pour l'ensemble des six localités, la concentration en calcium a varié de 3,09 à $4,93 \mathrm{cmol}^{+} / \mathrm{kg}$. Lorsqu'on se réfère à la norme (Ballot et al., 2016), 63\% des exploitations ont une faible concentration en calcium. Par rapport au magnésium, la concentration a varié de 0,65 à $1,76 \mathrm{cmol}^{+} / \mathrm{kg}$ avec $17 \%$ de valeurs en dessous du seuil critique $(0,5$ $\mathrm{cmol}^{+} / \mathrm{kg}$ ). S'agissant du potassium échangeable, les concentrations ont varié de 0,13 à $0,23 \mathrm{cmol}^{+} / \mathrm{kg}$ dont $77 \%$ en dessous du seuil critique $\left(0,2 \mathrm{cmol}^{+} / \mathrm{kg}\right)$. Même à de forte proportion de faibles concentrations, le complexe argilo-humique est essentiellement dominé par le calcium. Il a saturé le complexe à plus de $70 \%$. Cette richesse du complexe argilo-humique en calcium peut expliquer le caractère peu acide des sols. L'amélioration du taux de matière organique dans ces sols contribuerait à améliorer la fraction fine du complexe et par conséquent, l'amélioration de la fixation du calcium.

Le taux de saturation a varié de 62 à 91,8\%. La plupart des sols sont moyennement à très saturés en cations échangeables $\left(\mathrm{Ca}^{2+}\right.$, $\left.\mathrm{Mg}^{2+}, \mathrm{K}^{+}\right)$. Malgré ces bons taux de saturation, il est important de souligner que les concentrations des cations sont faibles. Ceci est lié à la qualité du complexe argilo- 
humique qui est pauvre en matière organique, et par conséquent, en humus. Ce dernier est déterminant dans la formation de la CEC (quantité de charges négatives par unité de masse d'un sol) qui augmente avec le taux d'humus dans le sol. Il est connu que seules les argiles et l'humus, par leurs propriétés colloïdales, peuvent développer des charges importantes à leur surface (Brady et Weil, 2002). Etant donné que la quantité et la qualité des argiles dans les sols sont dépendantes des conditions naturelles d'altération, c'est surtout l'augmentation ou la diminution de la teneur en matière organique qui influence la variation de la CEC pour les sols cultivés.

\section{Phosphore assimilable}

La teneur des sols en phosphore assimilable est très faible à faible dans une proportion de $80 \%$ des exploitations. Ce constat serait lié aux faibles taux de matière organique observés dans les sols. De nombreux auteurs ont souligné l'importance de la matière organique dans la disponibilité du phosphore assimilable (Tumer, 2008) et du potassium échangeable (Ballot et al., 2016). La matière organique constitue une source importante de phosphore sous forme organique mais également contribue au stockage réversible des éléments nutritifs par l'intermédiaire de la minéralisation/immobilisation par les microorganismes (Bertrand et Guigou, 2000). La décomposition de la matière organique libère certains acides organiques (Rabetokotany, 2008) qui ont la capacité de former les complexes Fer-Phosphore ou Aluminium-Phosphore en complexes plus stables à la surface des particules d'argile. Les apports de matières organiques s'avèrent indispensables dans la synthèse des enzymes phosphatases, ce qui permet de mobiliser le phosphore organique provenant notamment des apports de matières organiques (Tumer, 2008). Un enrichissement du sol en matière organique augmente la contribution du phosphore organique à la dynamique du phosphore (Parent et Khiari, 2003). L'humus présente des sites de faible énergie de liaison pour le phosphore rendant sa mise en disponibilité plus facile. Ainsi, la matière organique du sol joue un rôle crucial dans la disponibilité du phosphore pour les plantes cultivées.

\section{Conclusion}

$\mathrm{Au}$ terme de l'étude dont l'objectif était d'évaluer les paramètres physicochimiques des sols pour une gestion durable des exploitations agricoles dans les systèmes de culture à base du coton au Bénin, il a été révélé un état de dégradation de la plupart des sols. Les paramètres de fertilité sont largement bas à très bas avec un accent particulier pour la matière organique. La valeur de la CEC et des cations échangeables sont largement bas, et ne peuvent pas valoriser les apports d'engrais minéraux. Si on sait que la plupart des sols ferrugineux tropicaux sont pauvres en argile, et contiennent surtout la kaolinite à faible pouvoir de fixation des cartions échangeables, l'humus qui provient de la minéralisation de la matière organique est excellent dans la formation du complexe argilo-humique et dans la fixation des cations. Les très bas taux de matière organique peuvent être corrigés à travers la bonne valorisation des résidus de récolte et la production locale de la matière organique qui est le socle de l'amélioration et du maintien du niveau de la fertilité des sols, et par conséquent de l'amélioration des rendements des cultures qui est le gage d'une sécurité alimentaire sûre.

\section{CONFLITS D'INTERETS}

Les auteurs déclarent qu'il n'existe aucun conflit d'intérêts pour cet article.

\section{CONTRIBUTIONS DES AUTEURS}

IA a réalisé l'étude de terrain et le suivi des analyses de sol. GD et CEA ont supervisé les travaux de prélèvement de sol. Tous les auteurs ont contribué à la rédaction et la correction du manuscrit.

\section{REMERCIEMENTS}

Les auteurs remercient le Centre de Recherches Agricoles Coton et Fibres (CRA$\mathrm{CF})$ et l'Institut National des Recherches 
Agricoles du Bénin (INRAB) pour le soutien financier et matériel.

\section{REFERENCES}

Aho E, Capo-Chichi G. 2017. Le coton africain entre pratiques modernes et traditionnelles: le changement de paradigme d'une économie sociale en Afrique de l'Ouest. Bull. Rech. Agron. Bénin. Numéro Spécial Développement Agricole Durable. DOI : http://www.slire.net \&http://www.inrab.org

Alexandre M, Hansrudolf $\mathrm{O}$, Raphaël C, Vincent B, Sokrat S. 2012. Effet à long terme des engrais organiques sur les propriétés du sol. Rech. Agron. Suisse, 3(3) : 148-155.

Annabi M, Bahri H, Latiri K. 2009. Statut organique et respiration microbienne des sols du nord de la Tunisie. Biotechnol. Agron. Soc. Environ., 13(3): 401-408.

Azontondé A, Igué M, Dagbénombakin G. 2009. Carte de fertilité des sols du Bénin par zone agro-écologique du Bénin. Rapport de consultation pour le compte d'Afrique Etude, Bénin, 128.

Ballot CSA, Mawussi G, Atakpama W, Moita-Nassy M, Yangakola TM, Zinga I, Silla S, Kperkouma W, Dercon G, Komlan B, Koffi A. 2016. Caractérisation physico- chimique des sols en vue de l'amélioration de la productivité du manioc (Manihot esculenta Crantz) dans la région de Damara au centre-sud de Centrafrique. Agronomie Africaine, 28 (1) : 9-23.

Banque Mondiale. 2016. Notes de politiques pour la nouvelle administration béninoise. $145 \mathrm{p}$.

Bassala JPO, M'Biandoun M, Ekorong, JA, Asfom P. 2008. Evolution de la fertilité des sols dans un système cotonniercéréales au Nord Cameroun: diagnostic et perspectives. SOMMAIRE/INHOUD/SUMARIO, 26(4) : 240-245.
Batamoussi M, Moumouni I, Tokoré Orou Méré SB. 2015. Contribution à l'amélioration des pratiques paysannes de production durable de coton (Gossypium hirsutum) au Bénin cas de la commune de Banikoara. Int. J. Biol. Chem. Sci., 9(5): 2401-2413. DOI: http://dx.doi.org/10.4314/ijbcs.v9i5.12

Bertrand R, Gigou J. 2000. La Fertilité des Sols Tropicaux $\left(\mathrm{N}^{\mathrm{o}} .40\right)$. Maisonneuve et Larose: Paris; 397 p.

Brady NC, Weil RR. 2002. The Nature and Properties of Soils (13th edn). Upper Saddle River, NJ, Pearson Education Inc: USA; $960 \mathrm{p}$.

Coulibaly K, Vall E, Autray P, Nacro HB, Sedogo M. 2012. Effets de la culture permanente coton-maïs sur l'évolution d'indicateurs de fertilité des sols de l'Ouest du Burkina Faso. Int. J. Biol. Chem. Sci., 6(3): 1069-1080. DOI: http://dx.doi.org/10.4314/ijbcs.v6i3.13

Dagbenonbakin G, Burkhardt J, Goldbach HE. 2002. Bilan des nutriments dans les 8 systèmes de production du bassin versant de l’Ouémé. In: Actes de la a scarce conférence: "The efficient management of water resources in the Ouémé Catchment - Water as resource in Benin: Identification and analysis of influencing factors, Cotonou $20 \quad-21$ mars, 2002, IMPETUS, Direction Générale de l'Hydraulique, Bénin.

Dagbenonbakin G. 2005. Productivity and Water Use Efficiency of Important Crops in the 9 Upper Oueme Catchment: Influence of Nutrient Limitations, Nutrient Balances and Soil Fertility. University of Bonn: Germany.

Hougni A, Imorou L, Dagoudo A, ZoumarouWallis N. 2016. Caractérisation agromorphologique de variétés de Cotonnier (Gossypium Hirsutum) pour une régionalisation économique pour la production du coton au Bénin. Europ. Scient. J., 12(36): 1857-7881. DOI : http://dx.doi.org/10.19044/esj.2016.v12n $36 \mathrm{p} 210$ 
Igué AM, Agossou V, Ogouvidé FT. 2008. Influence des systèmes d'exploitation agricole sur l'intensité de la dégradation des terres dans le Département des Collines au Bénin. Bull. Rech. Agron. Bénin, 61 : 39-51.

Koulibaly B, Traoré O, Dakuo D, Zombré PN, Bondé D. 2010. Effets de la gestion des résidus de récolte sur les rendements et les bilans culturaux d'une rotation cotonnier - maïs. Tropicultura, 28(3) : 184-189.

Igué AM, Saidou A, Adjanohoun A, Ezui G, Attiogbe P, Kpagbin Gotoechan H, Youl S, Pare T, Balogoun I, Ouedrago J, Dossa E, Mando A, Sogbedji JM. 2013. Evaluation de la fertilité des sols au sud et centre du Bénin. Bull. Rech. Agron. Bénin, numéro spécial, Fertilisation du maïs, 12-23.

Igue MA, Oga AC, Balogoun I, Saidou A, Ezui G, Youl S, Sogbedji JM. 2016. Détermination Des Formules D'engrais Minéraux Et Organiques Sur Deux Types De Sols Pour Une Meilleure Productivité De Maïs (Zea mays 1.) Dans La Commune De Banikoara (Nord-Est Du Bénin). Euro. Scient. J., 12(30): 362-377. DOI :

http://dx.doi.org/10.19044/esj.2016.v12n 30p362.

Koull N, Halilat MT. 2016. Effets de la matière organique sur les propriétés physiques et chimiques des sols sableux de la région d'Ouargla (Algérie). Et. Gest. Sols, 23.

Koulibaly B, Traoré O, Dakuo D, Lalsaga R, Lompo F, Zombré PN. 2014. Acidification des sols ferrugineux et ferrallitiques dans les systèmes de production cotonnière au Burkina

Faso. Int. J. Biol. Chem. Sci., 8(6): 28792890.

DOI: http://dx.doi.org/10.4314/ijbcs.v8i6.44

Mbonigaba JJM, Nzeyimana I, Bucagu C, Culot M. 2009. Caractérisation physique, chimique et microbiologique de trois sols acides tropicaux du Rwanda sous jachères naturelles : contraintes à leur productivité. Rwanda J., 17(1): 34-63.

M'Biandoun M, Douzet JM. 2000. Evolution de la fertilité des sols sous culture dans les aménagements en courbe de niveau réalisés en 1996. Rapport d'analyse des résultats, IRAD-DPGT-

SODECOTON. Garoua, Nord Cameroun.

Menzies NW. 2003. Toxic Elements in Acidic Soils: chemistry and measurement. In Hand Book of Soil Acidity, Rengel Z Z (ed). Marcel Dekker: New-York, USA; 267-296.

Mulaji C, Disa-Disa P, Kibal I, Culot M. 2016. Diagnostic de l'état agropédologique des sols acides de la province de Kinshasa en république démocratique du Congo (RDC). Comptes Rendus Chimie, 19(7) : 820826. DOI : http://dx.doi.org/10.1016/j.crci.2015.08. 010

Ouandaogo N, Ouattara B, Bouinzemwendé M, Pouya PM, 2016. Effets des fumures organo-minérales et des rotations culturales sur la qualité des sols. Int. J. Biol. Chem. Sci., 10(2): 904-918. DOI : http://dx.doi.org/10.4314/ijbcs.v10i2.37

Oumarou. 2012. Capital Sol et arrangements institutionnels dans les agrosystèmes du Nord-Cameroun. Thèse de doctorat, centre international d'études supérieures en science agronomiques, Montpellier Supagro. $203 \mathrm{p}$

Parent LE, Khiari L 2003. Nitrogen and phosphorus indicators of organic soil quality. Parent.

Pernes-Debuyser A, Tessier D. 2002. Influence du $\mathrm{pH}$ sur les propriétés des sols: l'essai de longue durée des 42 parcelles à Versailles. Rev. Sc. eau/J. Wat. Sc. 15: 27-39. https://doi.org/10.7202/705484ar

Pouya MB, Bonzi M, Gnankambary Z, Traoré K, Ouédraogo JS, Somé AN, Sédogo MP. 2013. Pratiques actuelles de gestion de la fertilité des sols et leurs effets sur la 
production du cotonnier et sur le sol dans les exploitations cotonnières du Centre et de l'Ouest du Burkina Faso. Cah. Agric., 22(4) : 282-292.

Rabetokotany R. 2007. Effets d'une fertilisation phosphatée par le Bat Guano et Triple Super Phosphate sur un sol ferralitique malgache. Cas de Ferralsol de «Tanety » sis à Lazaina. Mémoire de DEA en Chimie, Université d'Antananarivo, p. 87

Tchabi V, Azocli D, Biaou GD, 2012. Effet de différentes doses de bouse de vache sur le rendement de la laitue (Lactuca sativa L.) à Tchatchou au Bénin. Int. J. Biol. Chem. Sci., 6(6): 5078-5084. DOI: http://dx.doi.org/10.4314/ijbcs.v6i6.26

Ton P. 2004. La production du coton au Bénin. Projet d'analyse d'une spéculation agricole par pays, financé par le programme "Renforcement des capacités commerciales" de la F.I.P.A (Fédération Internationale des Producteurs Agricoles). $52 \mathrm{p}$.

Turner BL. 2008. Resource partitioning for soil phosphorus: a hypothesis. J. Ecol., 96: 698-702.

Walkley A, Black IA. 1934. An examination of the Degtjareff method for determining soil organic matter, and a proposed modification of the chromic acid titration method. Soil Science, 37(1): 29-38.

Yemefack M, Nounamo L, Njomgang R, Bilong P. 2004. Influence des pratiques agricoles sur la teneur en argile et autres propriétés agronomiques d'un sol ferrallitique au sud Cameroun. Tropicultura, 22(1) : 3-10. 